\title{
The Role of CD40 in Allergic Rhinitis and Airway Remodelling
}

\author{
Ke-Jia Cheng $\mathbb{D}$, Min-Li Zhou $\mathbb{D}$, Yong-Cai Liu $\mathbb{D}$, Chen Wang $\mathbb{D}$, and Ying-Ying Xu $\mathbb{D}$ \\ Department of Otolaryngology, The First Affiliated Hospital, School of Medicine, Zhejiang University, Hangzhou, China \\ Correspondence should be addressed to Ying-Ying Xu; 1184031@zju.edu.cn
}

Received 26 October 2020; Revised 7 March 2021; Accepted 4 April 2021; Published 23 April 2021

Academic Editor: Paola Migliorini

Copyright (c) $2021 \mathrm{Ke}$-Jia Cheng et al. This is an open access article distributed under the Creative Commons Attribution License, which permits unrestricted use, distribution, and reproduction in any medium, provided the original work is properly cited.

\begin{abstract}
Background. Allergic rhinitis (AR) affects millions of people and is lack of effective treatment. CD40 is an important costimulatory molecule in immunity. However, few studies have focused on the role of CD40 in AR. Methods. In this study, we built mouse model of chronic AR. The mice were divided into the AR, control, intravenous CD40 siRNA, and nasal CD40 siRNA groups ( $n=6$ each). We detected OVA-sIgE, IL-4, IL-5, IL-13, IL-10, IFN- $\gamma$, and TGF- $\beta$ levels in serum and supernatant by ELISA, CD40 ${ }^{+}$splenic DCs, and Foxp $3^{+}$Tregs by flow cytometry and CD40 mRNA by RT ${ }^{2}$-PCR. We also used PAS and MT stains to assess tissue remodelling. Results. (1) The OVA-sIgE, IL-4, IL-5, and IL-13 levels in the serum or supernatant of nasal septal membrane of AR mice were significantly higher than control. After treated with CD40 siRNA, those indicators were significantly decreased. The IFN- $\gamma$, IL10 , and TGF- $\beta$ levels in AR mice were significantly lower than that in control and were increased by administration of CD40 siRNA. (2) AR mice had significantly fewer Foxp $3^{+}$Tregs in the spleen than control mice. After treated with CD40 siRNA, AR mice had significantly more Foxp3 ${ }^{+}$Tregs. (3) AR mice exhibited a significantly higher CD40 mRNA levels than control. Administration of CD40 siRNA significantly reduced the CD40 mRNA level. (4) The AR mice showed significantly greater collagen deposition than the control in MT staining. Applications of CD40 siRNA significantly reduced the collagen deposition in AR mice. Conclusion. CD40 siRNA therapy shows promise for chronic AR as it significantly attenuated allergic symptoms and Th2-related inflammation and upregulated Foxp $3^{+}$Tregs. CD40 plays a role in tissue remodelling in AR, which can be inhibited by CD40 siRNA application.
\end{abstract}

\section{Introduction}

Allergic rhinitis (AR) affects millions of people and is induced by immunoglobulin $\mathrm{E}$ (IgE). AR is a common disorder caused by a type 2 immune response to antigen as well as the recruitment and activation of mast cells, eosinophils, and basophils $[1,2]$. Type 2 inflammation promotes the activity of the cytokines IL-4, IL-5, and IL-13 [3]. IL-4 causes isotype class switching in B cells to produce IgE; IL-5 promotes eosinophil activation and recruitment; IL-13 triggers mucus hypersecretion and airway hypersensitivity $[4,5]$. AR can be divided into intermittent and persistent subtypes and is characterised by nasal congestion, rhinorrhoea, sneezing, and itching [6]. There is no effective treatment for $\mathrm{AR}$, which tends to recur and impair the quality of life of affected patients.

Airway remodelling is defined as any change in the composition, distribution, thickness, mass, volume, and number of structural components in the airway wall of patients compared with healthy individuals [7]. It usually manifests as goblet cell hyperplasia, basal membrane thickening, subepithelial fibrosis, hyperplasia and hypertrophy of smoothmuscle cells, and angiogenesis [8]. Airway remodelling is common in lower airway diseases, particularly asthma [9]. Asthma is difficult to treat, due in part to airway remodelling. Because of the difference in smooth muscle and blood sinus between the lower and upper airways, whether remodelling involves in AR is still controversial. Some researches have implied the existence of remodelling in AR $[10,11]$. Hence, we speculated that refractory AR might be linked to remodelling of the nasal mucosa.

CD40 is a type I membrane protein and a member of the tumour necrosis factor receptor (TNFR) superfamily [12]. It is expressed on B cells, dendritic cells (DCs), macrophages, monocytes, epithelial cells, endothelial cells, mesenchymal cells, and platelets. The CD40 pathway is an important costimulatory pathway in immunity. The interaction of CD40 and its ligand is required for effective adaptive and innate immune responses as it induces several immunostimulatory events, 
including the licensing of antigen-presenting cells (APCs), T cell activation, and B cell class switching [13]. CD40 has been linked to numerous diseases, including metabolic, neoplastic, autoimmune, cardiovascular, bowel, and allergic conditions. Moreover, CD40 is implicated in lower airway diseases, especially in allergy. However, few studies have focused on the role of CD40 in AR.

In the present study, we investigated the feasibility of CD40 small interfering RNA (siRNA) as a means of controlling $\mathrm{AR}$ and related airway remodelling in vivo. We found that nasal application of CD40 siRNA is effective against chronic AR and airway remodelling.

\section{Materials and Methods}

2.1. Ethics Statement. All mice were managed in accordance with protocols approved by the Research Ethics Committee of the First Affiliated Hospital, College of Medicine, Zhejiang University (Zhejiang, China).

2.2. Mouse Model of Chronic AR. Six-week-old adult BALB/c mice weighing 18-20g were purchased from Shanghai Animal Laboratories (Shanghai, China). The mice were divided into the AR, control, intravenous CD40 siRNA, and nasal CD40 siRNA groups ( $n=6$ each). AR mice were injected intraperitoneally with $10 \mu \mathrm{g}$ ovalbumin (OVA) and $4 \mathrm{mg}$ $\mathrm{Al}\left(\mathrm{OH}_{3}\right)$ on days 0 and 14 and challenged intranasally on days 29 to 35 with OVA $(600 \mu \mathrm{g})$; thereafter, the mice were challenged intranasally twice weekly for 8 weeks. Subsequently, sneezing and nasal rubbing movements were evaluated for $30 \mathrm{~min}$. Control mice underwent intranasal application of phosphate-buffered saline (PBS) only. The mice were sacrificed $24 \mathrm{~h}$ after the last challenge by intraperitoneal injection of excess pentobarbital, and the serum and spleen were collected for analysis. One side of the nasal septal membrane was collected for reverse transcription and real-time polymerase chain reaction ( $\mathrm{RT}^{2}$-PCR), and the other side was collected for histological assessment.

2.3. CD40 siRNA and Gene Silencing. The siRNA for silencing of murine CD40 (SI02739016 [FlexiTube siRNA]) was purchased from Qiagen (Qiagen Inc., Germantown, USA). Mice in the intravenous CD40 siRNA group were injected intravenously with $50 \mu \mathrm{g}$ of CD40 siRNA on days 27, 41, 55,69 , and 83 . Mice in the nasal CD40 siRNA group received $60 \mu \mathrm{g}$ CD40 siRNA intranasally on days $27,41,55,69$, and 83. Mice in the treatment and control groups received $60 \mu \mathrm{g}$ PBS intranasally at the same time points (Figure 1).

2.4. Measurement of OVA-sIgE and Inflammatory Cytokine Levels in Serum and Supernatant. Samples were allowed to clot in a serum separator tube for $30 \mathrm{~min}$, centrifuged for $15 \mathrm{~min}$ at approximately $1,000 \mathrm{rpm}$, and stored at $-20^{\circ} \mathrm{C}$. The nasal septal membrane was collected immediately after euthanasia. The specimens were thawed in saline $(0.1 \mathrm{~g}$ in $1 \mathrm{~mL}$ ) and processed in a tissue homogeniser. Next, the suspensions were centrifuged for $5 \mathrm{~min}$ at 3,000 rpm. The supernatants were collected and stored at $-20^{\circ} \mathrm{C}$ prior to analysis.

The levels of OVA-sIgE (Bio-Swamp, Wuhan, China), IL-5 (eBioscience, California, USA), and IFN- $\gamma$ (eBioscience,
California, USA) in the serum and supernatant were measured by enzyme-linked immunosorbent assay (ELISA) according to the manufacturer's instructions. IL-4 (eBioscience, California, USA), IL-13 (eBioscience, California, USA), IL-10 (eBioscience, California, USA), and TGF- $\beta$ (R\&D Systems, Minnesota, USA) levels were also detected by ELISA. In brief, the samples $(40 \mu \mathrm{L})$ were added to 96 -well plates, followed by the appropriate antibody $(10 \mu \mathrm{L})$. The plates were then closed and incubated for $30 \mathrm{~min}$ at $37^{\circ} \mathrm{C}$. Wash buffer was added to each well, the plates were allowed to stand for $30 \mathrm{~s}$, and the wash buffer was gently tapped out; this procedure was performed a total of five times. Next, $50 \mu \mathrm{L}$ of horseradish peroxidase- (HRP-) conjugate reagent were added to each well, except the blank well, and the plate was incubated and washed as before. Chromogen solution A and chromogen solution B ( $50 \mu \mathrm{L}$ each) were added to each well, and the plates were gently mixed and incubated for $15 \mathrm{~min}$ at $37^{\circ} \mathrm{C}$. Next, $50 \mu \mathrm{L}$ of stop solution were added to each well, and the optical density at $450 \mathrm{~nm}$ was read within $15 \mathrm{~min}$. A standard curve was generated to enable quantification.

2.5. Preparation of Spleen Mononuclear Cell Suspensions. The minced spleen tissues of BALB/c mice were mixed with RMPI-1640 medium and centrifuged for $10 \mathrm{~min}$ at 3,000 rpm. The intermediate white film was next centrifuged in RMPI-1640 medium for $10 \mathrm{~min}$ at 3,000 rpm. Finally, red cells were removed using ACK lysis buffer, and the resulting spleen mononuclear cell (sMNC) suspensions were centrifuged in RMPI-1640 medium for $10 \mathrm{~min}$ at 3,000 rpm.

2.6. Flow Cytometry Analysis of $\mathrm{CD}^{+} 0^{+}$Splenic DCs and Forkhead Box P3 (Foxp3) ${ }^{+}$Tregs. sMNC suspensions were diluted with PBS to $10^{6}$ cells/mL. sMNCs $(100 \mu \mathrm{L})$ were incubated with $5 \mu \mathrm{L}$ of anti-CD4 and -CD25 mAbs (eBioscience) at $4^{\circ} \mathrm{C}$ in the dark for $60 \mathrm{~min}$. Then, we fixed and broken membrane. Next, sMNCs were added to $5 \mu \mathrm{L}$ of anti-Foxp3 $\mathrm{mAb}$ (eBioscience). Flow cytometric analysis of Foxp $3^{+}$Tregs was performed using a FACScan (Becton Dickinson, New Jersey, USA). We used $3 \mu \mathrm{L}$ of anti-CD40 and $1 \mu \mathrm{L}$ of anti$\mathrm{CD} 11 \mathrm{c}$ mAb (eBioscience) to analyse CD $40^{+}$DCs.

2.7. $R T^{2}-P C R$ for CD40. Total RNA was isolated from nasal septal membranes using TRIzol reagent (Invitrogen, California, USA) according to the manufacturer's protocol. To generate first-strand cDNA, samples were treated with DNase I to remove DNA and subjected to reverse transcription in reactions containing RNA-Primer Mix $(5 \mu \mathrm{L}), 25 \mathrm{mM}$ dNTPs $(1 \mu \mathrm{L})$, $25 \mathrm{U} / \mu \mathrm{L}$ RNase inhibitor $(1 \mu \mathrm{L}), 200 \mathrm{U} / \mu \mathrm{L}$ M-MLV reverse transcriptase $(1 \mu \mathrm{L})$, oligo $(1 \mu \mathrm{L})$, and DNase-free water $(4 \mu \mathrm{L})$.

The CD40 primers were sense $5^{\prime}$-CCCTGCGATGGTGT CTTTGC-3' and antisense $5^{\prime}$-TGGCTTGTCAGTCGGC TTCC- $3^{\prime}$. The primers for GAPDH (control) were sense $5^{\prime}$ -ATCACTGCCACCCAGAAG-3' and antisense $5^{\prime}$-TCCA CGACGGACACATTG-3'. Real-time PCR was performed at $95^{\circ} \mathrm{C}$ for $10 \mathrm{~min}, 95^{\circ} \mathrm{C}$ for $15 \mathrm{~s}, 60^{\circ} \mathrm{C}$ for $45 \mathrm{~s}$ ( $40 \mathrm{cycles}$ ), $95^{\circ} \mathrm{C}$ for $15 \mathrm{~s}, 60^{\circ} \mathrm{C}$ for $1 \mathrm{~min}, 95^{\circ} \mathrm{C}$ for $15 \mathrm{~s}$, and $60^{\circ} \mathrm{C}$ for $15 \mathrm{~s}$.

2.8. Assessment of Tissue Remodelling. Nasal septal membranes were inflated with $4 \%$ paraformaldehyde at $25 \mathrm{~cm}$ 


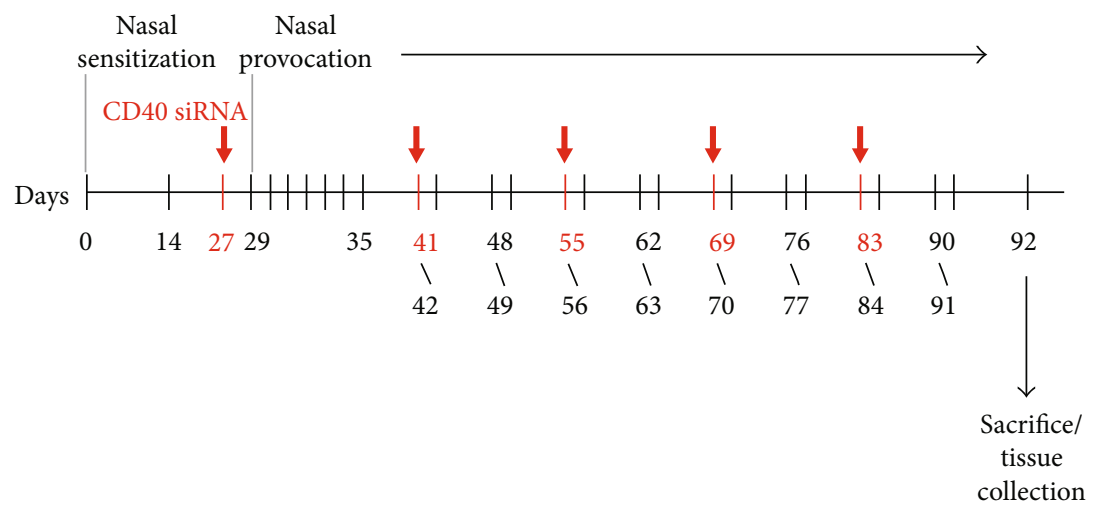

FIgURE 1: Mouse model of chronic AR and CD40 siRNA application.

$\mathrm{H}_{2} \mathrm{O}$ pressure. Paraffin-embedded sections $(4 \mu \mathrm{m})$ were stained with periodic acid-Schiff (PAS) to identify goblet cell hyperplasia, which was quantified in three selected areas at a 200-fold magnification. The number of goblet cells in the epithelium was separately assessed by two researchers, and the average values were calculated.

The sections were stained with Masson's trichrome (MT) to identify collagen deposition. As previous article described, the area of collagen deposition (blue staining) beneath the basement membrane in three regions was evaluated at a 200-fold magnification. Collagen deposition was evaluated as the percentage blue-stained area in the nasal mucosa using the Image J software [5].

2.9. Statistical Analysis. Values are expressed as median (25$75 \%$ ) using the SPSS version 20.0 software. Differences in symptoms were analysed by Fisher's exact test. Differences between groups were evaluated with the Mann-Whitney $U$-test. A value of $P<0.05$ was considered indicative of statistical significance.

\section{Results}

3.1. CD40 siRNA Treatment Decreases Symptoms in AR Mice. After the last nasal challenge, AR, but not control, mice showed sneezing and nasal rubbing. There was significantly difference between AR and control group. One of the mice administered CD40 siRNA intravenously and nasally showed sneezing and nasal rubbing. There was significantly difference between CD40 siRNA and AR group.

3.2. CD40 siRNA Treatment Decreases the OVA-sIgE Level, Inhibits the Th2 Immune Response, and Promotes the Th1 Immune Response. Serum and supernatant levels of OVAsIgE, Th 2 cytokine IL-5, and Th1 cytokine IFN- $\gamma$ of AR mice were measured by ELISA. The OVA-sIgE and IL-5 levels in the serum and supernatant of AR mice were significantly higher than those in control mice. The OVA-sIgE and IL-5 levels in mice treated with CD40 siRNA were lower than those in AR mice. Mice treated nasally with CD40 siRNA produced significantly less OVA-sIgE than mice intravenously administered CD40 siRNA (Figures 2(a) and 2(b)). The IFN- $\gamma$ level in the serum and supernatant of AR mice was significantly lower than that in control mice and was increased by administration of CD40 siRNA. The IFN- $\gamma$ level in mice treated nasally with CD40 siRNA was significantly higher than that in mice treated intravenously (Figure 2(c)). Supernatant levels of IL- 4 , IL-13, IL-10, and TGF- $\beta$ were also measured by ELISA. The IL- 4 and IL-13 levels in the supernatant of AR mice were significantly increased compared to those in control. After treated with CD40 siRNA, the IL-4 and IL-13 levels in the supernatant were significantly reduced (Figures 2(d) and 2(e)). However, no difference of the IL-4 and IL-13 levels in the supernatant was observed between nasal and intravenous siRNA groups. In addition, the IL-10 and TGF- $\beta$ levels in the supernatant of AR mice were significantly decreased as compared with those in control. After treated with CD40 siRNA, the IL-10 and TGF- $\beta$ levels in the supernatant were significantly upregulated (Figures $2(\mathrm{f})$ and $2(\mathrm{~g})$ ). Similarly, no difference of the IL-10 and TGF- $\beta$ levels in the supernatant was observed between nasal and intravenous siRNA groups. These results suggest that CD40 siRNA inhibits the allergic and Th2 responses and promotes the Th1 response and immune tolerance. CD40 siRNA applied by nasal pathway may more powerful than intravenous pathway.

3.3. CD40 siRNA Treatment Inhibits $C D 40^{+}$DCs and Increases the Foxp $3^{+}$Treg Population in the Spleen. DCs are important APCs that express CD40 [14]. To assess the efficacy of gene silencing by CD40 siRNA in vivo, we investigated CD40 expression on DCs by flow cytometry (Table 1). The number of DCs in AR mice was higher than that in control mice but did not differ between AR and siRNA mice. This suggests that DCs play a role in the pathogenesis of $A R$, and that this role is independent of that played by CD40.

CD40 siRNA treatment suppressed CD40 expression by DCs. There was no significant difference in CD40 expression on DCs between mice treated with the CD40 siRNA intravenously and nasally (Figures 3(a) and 3(b)) Therefore, the CD40 siRNA knocked down CD40 expression by DCs in vivo.

Tregs contribute to active immune suppression [15]. We examined the effect of CD40 siRNA on Treg generation in vivo by analysing $\mathrm{CD} 4^{+} \mathrm{CD} 25^{+}$Foxp $^{+}$cells in sMNCs by flow cytometry (Table 1). AR mice had significantly fewer 


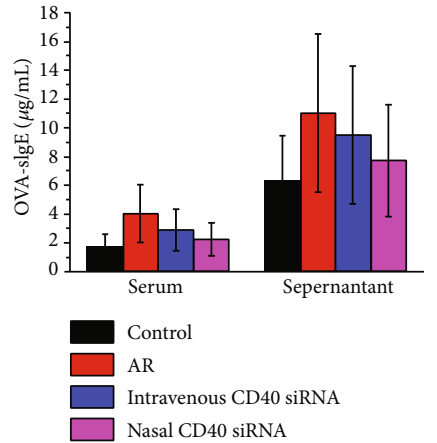

(a)

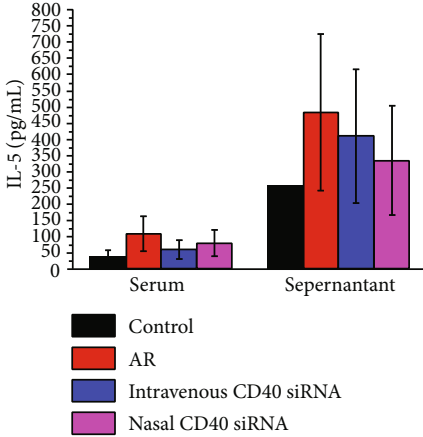

(b)

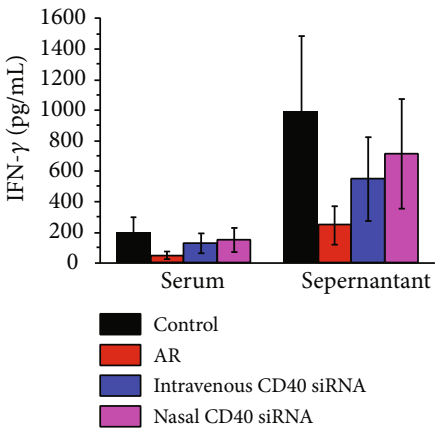

(c)

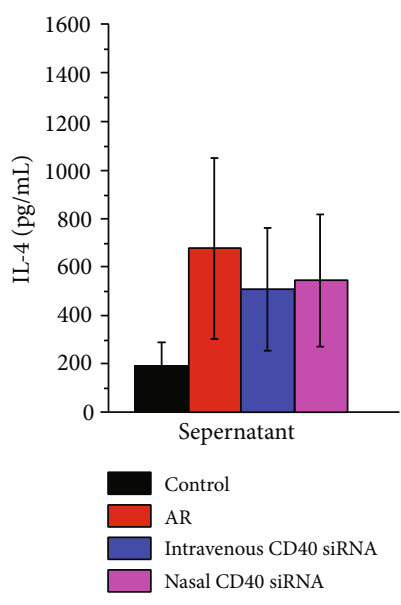

(d)

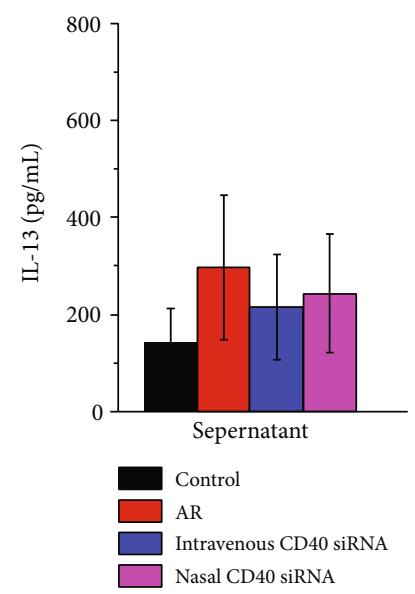

(e)

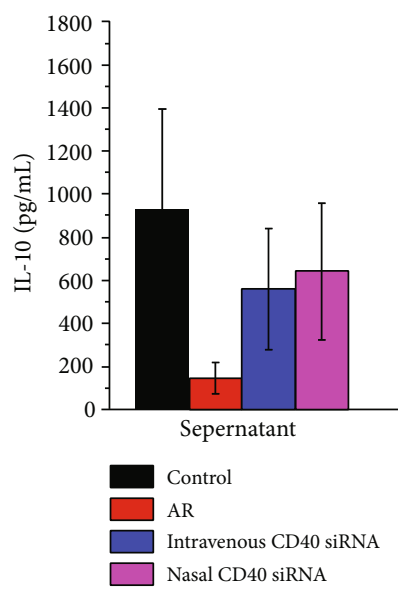

(f)

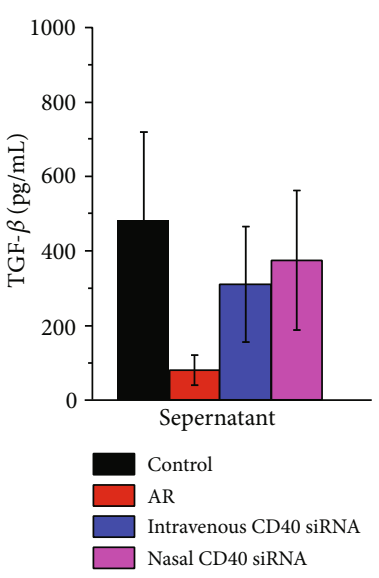

(g)

FIgURE 2: (a) Serum and supernatant levels of OVA-sIgE were measured by ELISA. AR mice produced significantly more OVA-sIgE than control. OVA-sIgE level in mice treated with CD40 siRNA was lower than AR mice. Mice treated nasally with CD40 siRNA produced significantly less OVA-sIgE than mice given intravenously CD40 siRNA. (b) Serum and supernatant levels of IL-5 were measured by ELISA. AR mice produced significantly more IL-5 than control. IL-5 level in mice treated with CD40 siRNA was lower than AR mice. (c) Serum and supernatant levels of IFN- $\gamma$ were measured by ELISA. AR mice produced significantly less IFN- $\gamma$ than control. IFN- $\gamma$ level in mice treated with CD40 siRNA was higher than AR mice. Mice treated nasally with CD40 siRNA produced significantly more IFN- $\gamma$ than mice given intravenously CD40 siRNA. (d) Supernatant levels of IL- 4 were measured by ELISA. AR mice produced significantly more IL-4 than control. IL-4 level in mice treated with CD40 siRNA was lower than AR mice. (e) Supernatant levels of IL-13 were measured by ELISA. AR mice produced significantly more IL-13 than control. IL-13 level in mice treated with CD40 siRNA was lower than AR mice. (f) Supernatant levels of IL-10 were measured by ELISA. AR mice produced significantly less IL-10 than control. IL-10 level in mice treated with CD40 siRNA was higher than AR mice. (g) Supernatant levels of TGF- $\beta$ were measured by ELISA. AR mice produced significantly less TGF- $\beta$ than control. TGF- $\beta$ level in mice treated with CD40 siRNA was higher than AR mice. $P$ values $<0.05$ were considered as significance.

TABLE 1: The percentage of DCs, CD40 DCs, and Tregs in the spleen.

\begin{tabular}{lcccc}
\hline Group & Control & AR & Intravenous CD40 siRNA & Nasal CD40 siRNA \\
\hline DCs (\%) & $69.150(65.950-75.925)$ & $84.750(73.275-88.725)$ & $73.450(70.400-84.375)$ & $75.850(69.550-84.625)$ \\
CD40 ${ }^{+}$DCs (\%) & $15.818(9.232-22.754)$ & $33.087(21.737-38.601)$ & $16.121(10.149-27.951)$ & $15.539(9.273-29.475)$ \\
Tregs (\%) & $4.803(4.249-7.042)$ & $0.687(0.373-3.035)$ & $2.075(1.490-2.814)$ & $3.728(2.142-4.289)$ \\
\hline
\end{tabular}

Foxp $3^{+}$Tregs than control mice. Mice treated with CD40 siRNA nasally had significantly more Foxp3 ${ }^{+}$Tregs than AR mice. There was no significant difference in the number of Foxp $3^{+}$Tregs between mice treated intravenously with CD40 siRNA and AR mice (Figures 3(a) and 3(c)). These findings implicate downregulation of Foxp $3^{+}$Tregs in the pathogenesis of AR and suggest that nasal administration of CD40 siRNA increases the splenic Foxp $3^{+}$Treg population.
3.4. CD40 siRNA Treatment Inhibits CD40 Expression in Nasal Tissues. The effect of CD40 siRNA on the CD40 mRNA level was evaluated by $\mathrm{RT}^{2}$-PCR. AR mice exhibited a significantly higher CD40 mRNA levels than control mice. Intravenous or nasal administration of CD40 siRNA significantly reduced the CD40 mRNA level. The CD40 mRNA level of mice treated with CD40 siRNA nasally was significantly lower than that of mice who received the CD40 siRNA 


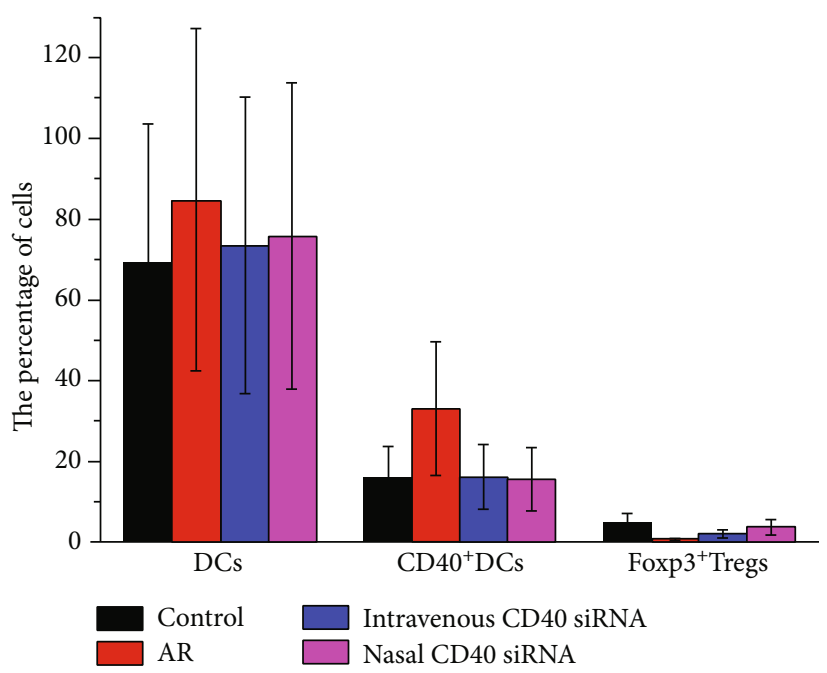

(a)

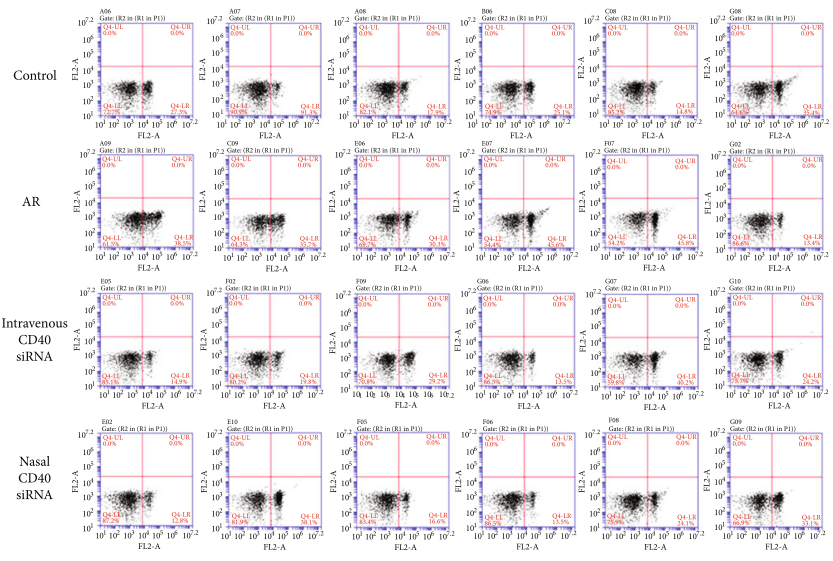

(b)

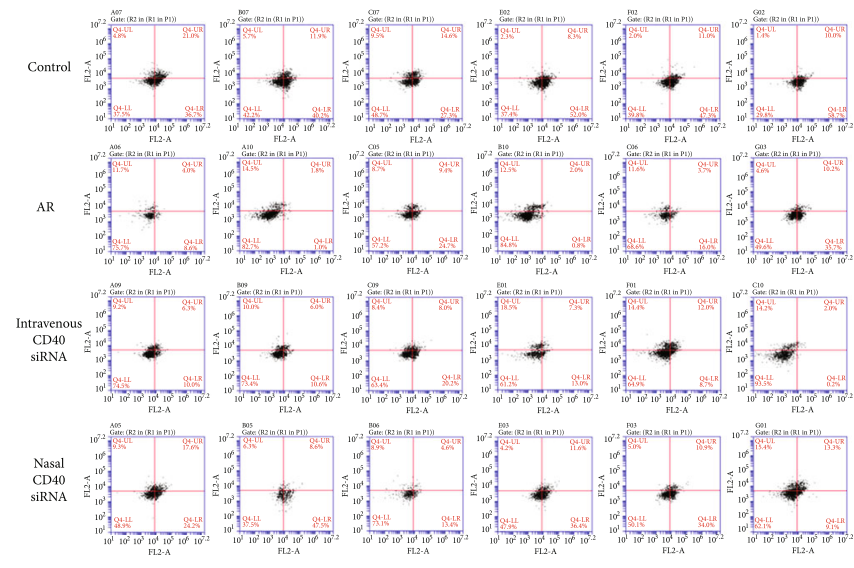

(c)

Figure 3: (a) CD40 ${ }^{+}$DCs and Foxp3 ${ }^{+}$Tregs of the spleen in control, AR, intravenous CD40 siRNA, and nasal CD40 siRNA groups were detected by flow cytometry. CD40 expression on DCs in AR mice was higher than control group. There was no difference between AR and siRNA groups. CD40 siRNA treatment resulted in inhibition of CD40 expression on DCs. There was no significant difference between intravenous and nasal siRNA group. AR mice shown significantly less Foxp $3^{+}$Tregs, compared with control mice. Mice nasally treated with CD40 siRNA had significantly more Foxp $3^{+}$Tregs, compared with AR mice. There was no significant difference between intravenous CD40 siRNA and AR group. $P$ values $<0.05$ were considered as significance. (b) $\mathrm{CD} 40^{+} \mathrm{DCs}$ of the spleen in control, AR, intravenous CD40 siRNA, and nasal CD40 siRNA groups were detected by flow cytometry. (c) Foxp $3^{+}$Tregs of the spleen in control, AR, intravenous CD40 siRNA, and nasal CD40 siRNA groups were detected by flow cytometry.

intravenously (Figure 4). This suggests that CD40 plays a role in the pathogenesis of AR, and that CD40 siRNA suppressed CD40 expression in vivo.

3.5. PAS and MT Staining. Tissue remodelling primarily involves goblet cell hyperplasia and collagen deposition, which are common in lower-airway inflammation, such as asthma [16]. However, whether tissue remodelling occurs in $\mathrm{AR}$ is controversial. We enumerated goblet cells by PAS staining and explored collagen deposition by MT staining (Table 2). There was no significant difference in the number of goblet cells among the AR, CD40 siRNA-treated, and control mice (Figures 5(a) and 5(c)). The AR mice showed significantly greater collagen deposition than the control mice. Also, mice treated nasally or intravenously with the
CD40 siRNA showed significantly less collagen deposition than did the AR mice (Figures 5(b) and 5(d)). These results suggest that tissue remodelling in AR manifests mainly as collagen deposition that it is related to the CD40 pathway, and that it can be inhibited by CD 40 siRNA treatment.

\section{Discussion}

$\mathrm{AR}$, which is Th2-mediated, is an eosinophilic allergic inflammation of the nasal membranes. AR patients have elevated IgE levels, eosinophil infiltration, and type 2 cytokine production [17]. However, the pathogenesis of AR is unclear. Until now, AR mice model usually use an acute model. In the present study, we developed a mouse model of chronic AR in an attempt to more accurately reflect naturally occurring AR. 


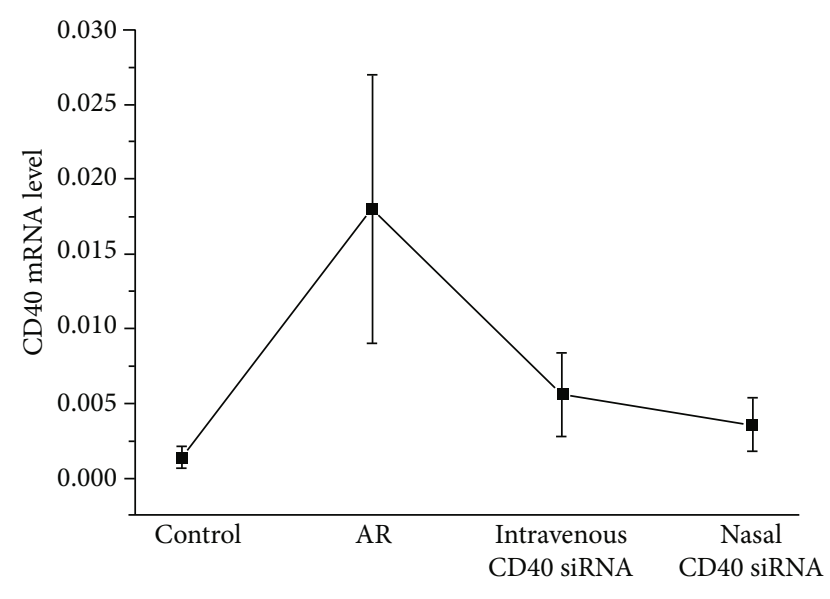

FIGURE 4: The mRNA level of CD40 in nasal tissues was detected by $\mathrm{RT}^{2}$-PCR. AR mice expressed significantly higher CD40 mRNA compared with control group. CD40 mRNA was significantly reduced in the mice treated with CD40 siRNA, both by intravenous and nasal methods. The mice treated with nasal CD40 siRNA expressed significantly lower CD40 mRNA than mice that received intravenous CD40 siRNA. $P$ values $<0.05$ were considered as significance.

In this study, we found that the levels of OVA-sIgE, IL-4, IL-5, and IL-13 in the serum or supernatant were higher in AR mice than in control mice. Meanwhile, the serum and supernatant IFN- $\gamma$ levels were lower in AR mice. These findings suggest that AR is mainly mediated by Th2 inflammation, which is in accordance with a previous report [18].

The CD40 pathway is an important costimulatory pathway in immunity. The CD40-CD40L interaction leads to several immunostimulatory events, including maturation of APCs, $\mathrm{T}$ cell activation, and B cell class switching [12]. Dysfunction of the CD40 pathway is implicated in many diseases, such as neoplastic, allergic, autoimmune, cardiovascular, and bowel disorders. CD40 also plays an important role in allergic inflammation of the lower airway [19-22]. CD40 signalling is important for OVA-induced eosinophilia and the production of IL-4, IL-5, and IL-13 in a mouse model of allergic asthma [19]. CD40 improves the sensitivity of related cells and promotes the production of proinflammatory cytokines after postexercise bronchoconstriction in patients with allergic asthma [20]. Moreover, CD40 induces the expression of numerous signalling molecules associated with airway inflammation and remodelling in allergic asthma [21]. CD40 gene polymorphisms may also influence IgE production in patients with asthma by modulating CD40 expression on B cells [22].

However, there are few publications to show the role of CD40 in AR. Production of specific IgE, IL-4, and IL-5 was reduced in $\mathrm{CD}_{40}{ }^{-/}$and Schistosoma mansoni egg antigeninduced AR mouse models [23]. IgE secretion by B cells was inhibited by promoting the combination of CD40L and IL-4 in patients with AR [24]. Recently, siRNA is used in studies of the role of CD40 in allergic inflammation. siRNAmediated RNA interference suppresses gene expression in vitro and in vivo [25] and is effective and long-lasting
[26]. Systemic administration of CD40 siRNA reportedly inhibits allergic responses and symptoms in vitro and in vivo $[27,28]$. CD40-silenced DCs have been shown to suppress OVA-induced allergy [29]. Local delivery of siRNA enables use of a lower dose and has a lower incidence of side effects. Therefore, we assessed intravenous and nasal applications of a CD40 siRNA. In this study, intravenous and nasal applications of CD40 siRNA significantly reduced the CD40 mRNA levels in nasal tissues, suggesting silencing of the CD40 gene. Also, the CD40 level differed between AR and control mice, and CD40 siRNA treatment reduced sneezing and nasal rubbing movements by the mice. In addition, CD40 siRNA treatment decreased the OVA-sIgE, IL-4, IL-5, and IL-13 levels and increased the IFN- $\gamma$ level. These findings suggest that CD40 plays an important role in the pathogenesis of $\mathrm{AR}$, and that CD40 siRNA treatment restores the Th1/Th2 balance and inhibits allergic inflammation in AR.

DCs are present in the skin and mucosae [30], and mice have several subgroups of DCs [31]. In this study, we used $\mathrm{CD}_{11} \mathrm{c}^{+}$as the screening criterion of DCs by flow cytometry. DCs recognise and capture invading foreign antigens and pathogens and regulate the immune response by interacting with T cells. However, the role of DCs in AR is controversial. DCs may activate effector $\mathrm{T}$ cells to promote immune responses or induce Tregs to promote immune tolerance. It has been reported that the number of DCs in the nasal mucosa of patients with AR significantly increases after allergen challenge [32]. In contrast, one study showed that DC-induced generation of IL-10-producing Tregs in vivo is dependent on ICOSL/ICOS and results in immune tolerance [33]. Here, we showed that the percentage of DCs in the spleens of AR mice was higher than that in those of control mice, suggesting that DCs promote inflammation in AR. Also, CD40 siRNA treatment significantly reduced CD40 expression on DCs, confirming knockdown of CD40 expression.

Downstream factors in the CD40 pathway are crucial for the maturation of, and cytokine secretion by, DCs. Blockade of CD40-receptor-associated factor-6 impairs DC maturation [34]. Meanwhile, the CD40-CD40L signalling axis induces migration of DCs and reduces the number of Tregs [35]. However, in this study, inhibition of CD40 had no effect on the number of DCs; thus, further research on the relationship between CD40 and DCs is needed.

Treg cells are a CD $4^{+} \mathrm{T}$ cell subset that promotes selfimmune tolerance and maintenance of immune balance [36]. There are at least two subtypes of Tregs-natural and inducible Tregs [37]. The Foxp3 gene is a critical regulator in the development and function of Tregs. Downregulation of Foxp3 may play an important role in the onset of AR [38]. In this study, the AR mice generated significantly fewer Foxp3+ Tregs, IL-10, and TGF- $\beta$ levels than the control mice, which is in agreement with a previous report [39]. Indeed, CD40 siRNA has been shown to increase Treg numbers in allergic mice [27]. In this study, mice treated nasally, but not those treated intravenously, with CD40 siRNA had significantly more Foxp $3^{+}$Tregs than AR mice. Meanwhile, after treated with CD40 siRNA, the supernatant IL-10 and TGF- $\beta$ levels were significantly upregulated in AR mice. These results suggest that inhibition of CD40 reduces allergic 
TABLE 2: Histopathology results of tissue remodelling.

\begin{tabular}{|c|c|c|c|c|}
\hline Group & Control & $\mathrm{AR}$ & $\begin{array}{c}\text { Intravenous CD40 } \\
\text { siRNA }\end{array}$ & $\begin{array}{l}\text { Nasal CD40 } \\
\text { siRNA }\end{array}$ \\
\hline PAS staining (goblet cells number) & $75.70(35.75-88.25)$ & $55.00(42.50-98.75)$ & $73.00(51.50-109.50)$ & $53.00(41.50-65.50)$ \\
\hline $\begin{array}{l}\text { MT staining (percentage of } \\
\text { collagen deposition) }\end{array}$ & $7.269(6.429-8.285)$ & $19.369(15.760-21.596)$ & $8.521(7.565-10.088)$ & $9.306(8.305-10.247)$ \\
\hline
\end{tabular}

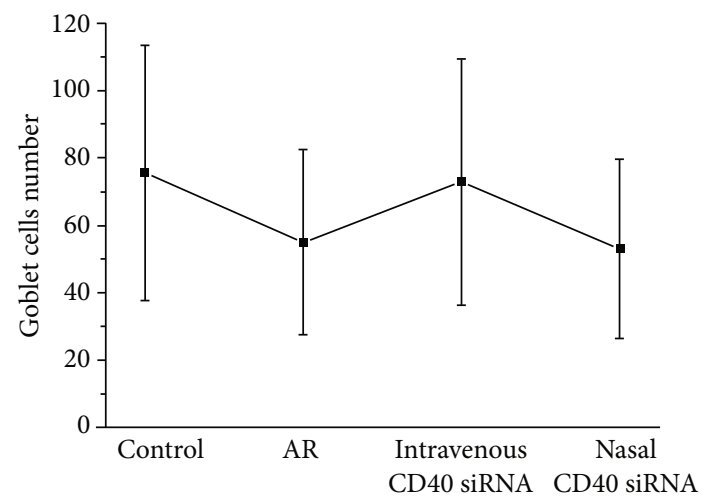

(a)

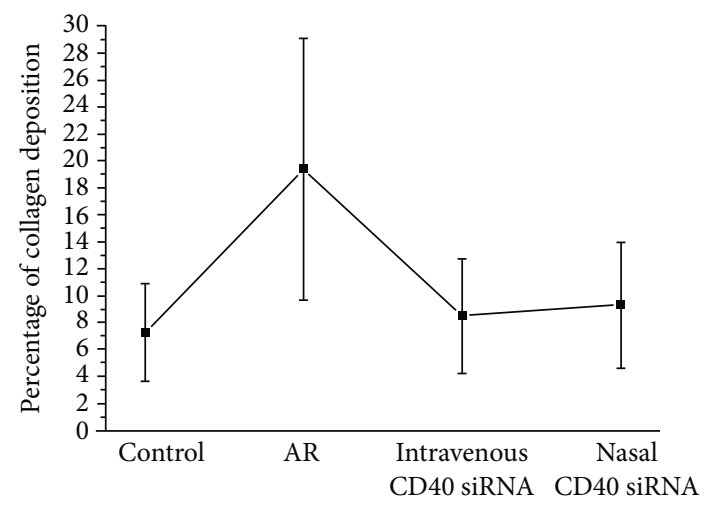

(b)

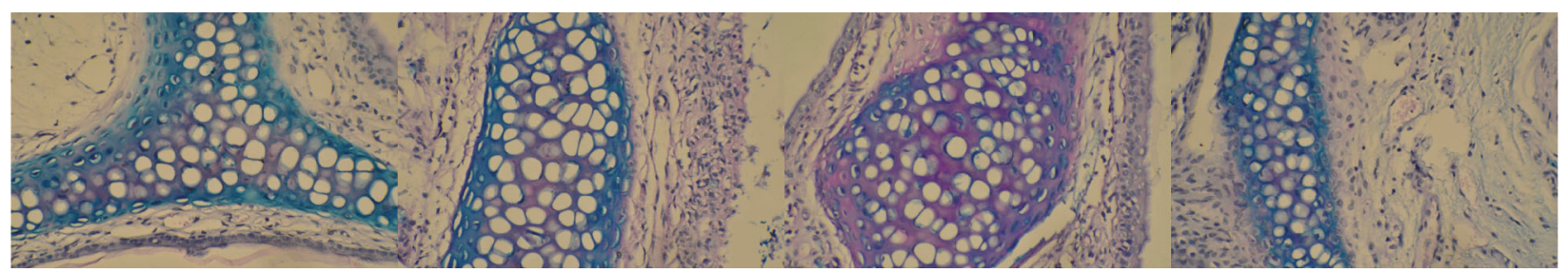

(c)

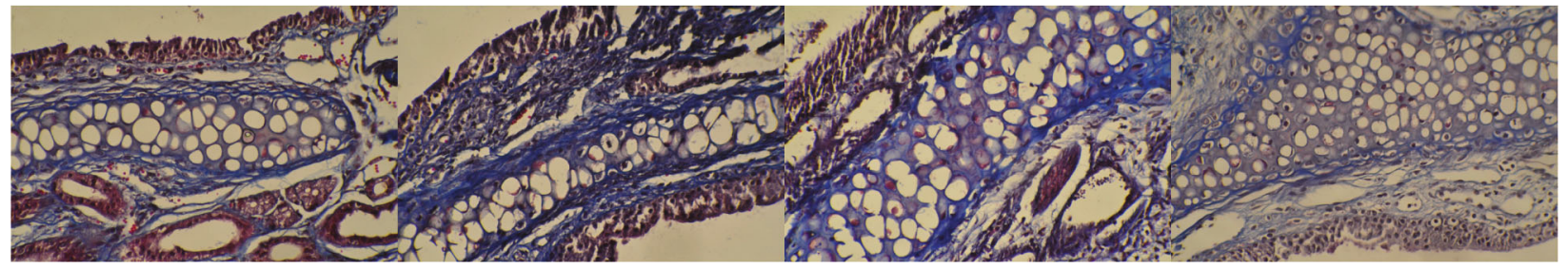

(d)

FIgure 5: (a) Goblet cell numbers of control, AR, intravenous CD40 siRNA, and nasal CD40 siRNA groups were detected by PAS staining. There was no significant difference in goblet cell numbers between AR, CD40 siRNA treatment, and control groups. All $P$ values $>0.05$. (b) Collagen deposition of control, AR, intravenous CD40 siRNA, and nasal CD40 siRNA groups was detected by MT staining. In this study, AR mice expressed significantly more collagen deposition, compared to control group. Mice treated nasally or intravenously with CD40 siRNA expressed significantly less collagen deposition, compared with AR mice. $P$ values $<0.05$ were considered as significance. (c) Goblet cell numbers of control, AR, intravenous CD40 siRNA, and nasal CD40 siRNA groups were detected by PAS staining. (d) Collagen deposition of control, AR, intravenous CD40 siRNA, and nasal CD40 siRNA groups was detected by MT staining.

responses, in part, by increasing the number of Tregs. We postulated that nasal application of CD40 siRNA was more powerful than intravenous.

Tissue remodelling is a common feature of allergic inflammation in the lower airway and can lead to irreversible airflow obstruction [40]. Rhinovirus infection can promote airway remodelling to trigger asthma [41]. However, whether tissue remodelling of the nasal cavity occurs in AR is uncertain. Nasal remodelling, including goblet cell hyperplasia and collagen deposition, has been reported to occur in AR mice $[10,11]$, whereas other studies have suggested that ani- mal models of AR involve microvascular remodelling of the nasal mucosa [42] and lung remodelling [43]. Additionally, remodelling of the nasal mucosa is reportedly more evident in AR patients with irreversible nasal obstruction [44]. In contrast, Eifan et al. found no histologic features of upperairway structural remodelling in patients with severe persistent AR [45]. In this study, the goblet cell count was similar in the AR and control mice, but the level of collagen deposition was higher in the AR mice. Thus, it is tempting to speculate that tissue remodelling in AR manifests mainly as collagen deposition. Further studies should focus on the roles 
of tissue remodelling-related cytokines in chronic AR. CD40 siRNA treatment significantly reduced collagen deposition in AR mice, suggesting that tissue remodelling in AR might be related to the CD40 pathway.

\section{Conclusions}

CD40 siRNA therapy shows promise for chronic AR as it significantly attenuated allergic symptoms and Th2-related inflammation and upregulated Foxp $3^{+}$Tregs and immune tolerance, which is in agreement with prior research [27, 28]. In this study, we make a new discovery that CD40 siRNA applied by nasal pathway can take roles in antiallergy in chronic AR process. We report here an important role for CD40 in tissue remodelling in AR, which can be inhibited by CD40 siRNA application.

\section{Data Availability}

The datasets used and/or analysed during the current study are available from the corresponding author on reasonable request.

\section{Conflicts of Interest}

The authors declare that they have no conflicts of interest.

\section{Acknowledgments}

This study was funded by National Natural Science Foundation of China (Grant No. 81770976). The English in this document has been checked by at least two professional editors, both native speakers of English. For a certificate, please see http://www.textcheck.com/certificate/8ZXUbq.

\section{References}

[1] M. Wang, Z. Gu, J. Yang, H. Zhao, and Z. Cao, "Changes among TGF- $\beta 1^{+}$Breg cells and helper $\mathrm{T}$ cell subsets in a murine model of allergic rhinitis with prolonged OVA challenge," International Immunopharmacology, vol. 69, pp. 347357, 2019.

[2] M. Caminati, D. L. Pham, D. Bagnasco, and G. W. Canonica, "Type 2 immunity in asthma," World Allergy Organization Journal, vol. 11, p. 13, 2018.

[3] B. N. Lambrecht, H. Hammad, and J. V. Fahy, "The cytokines of asthma," Immunity, vol. 50, no. 4, pp. 975-991, 2019.

[4] K. Sugita, C. Altunbulakli, H. Morita et al., "Human type 2 innate lymphoid cells disrupt skin keratinocyte tight junction barrier by IL-13," Allergy, vol. 74, no. 12, pp. 2534-2537, 2019.

[5] D. Russkamp, A. Aguilar-Pimentel, F. Alessandrini et al., "IL-4 receptor $\alpha$ blockade prevents sensitization and alters acute and long-lasting effects of allergen-specific immunotherapy of murine allergic asthma," Allergy, vol. 74, no. 8, pp. 15491560, 2019.

[6] M. Maurer and T. Zuberbier, "Under-treatment of rhinitis symptoms in Europe: findings from a cross-sectional questionnaire survey," Allergy, vol. 62, no. 9, pp. 1057-1063, 2007.

[7] N. Hirota and J. G. Martin, "Mechanisms of airway remodeling," Chest, vol. 144, no. 3, pp. 1026-1032, 2013.
[8] H. Fehrenbach, C. Wagner, and M. Wegmann, "Airway remodeling in asthma: what really matters," Cell and Tissue Research, vol. 367, no. 3, pp. 551-569, 2017.

[9] W. J. Ye, W. G. Xu, X. J. Guo et al., "Differences in airway remodeling and airway inflammation among moderatesevere asthma clinical phenotypes," Journal of Thoracic Disease, vol. 9, no. 9, pp. 2904-2914, 2017.

[10] Z. Q. Guo, W. Y. Dong, J. Xu et al., “T-helper type 1-T-helper type 2 shift and nasal remodeling after fine particulate matter exposure in a rat model of allergic rhinitis," American Journal of Rhinology \& Allergy, vol. 31, no. 3, pp. 148-155, 2017.

[11] H. Hong, S. Liao, F. Chen, Q. Yang, and D. Y. Wang, "Role of IL-25, IL-33, and TSLP in triggering united airway diseases toward type 2 inflammation," Allergy, vol. 75, no. 11, pp. 2794-2804, 2020.

[12] T. Kawabe, M. Matsushima, N. Hashimoto, K. Imaizumi, and Y. Hasegawa, "CD40/CD40 ligand interactions in immune responses and pulmonary immunity," Nagoya Journal of Medical Science, vol. 73, pp. 69-78, 2017.

[13] D. F. Pinelli and M. L. Ford, "Novel insights into antiCD40/CD154 immunotherapy in transplant tolerance," Immunotherapy, vol. 7, no. 4, pp. 399-410, 2015.

[14] H. Wang, C. Zhao, M. Zhang, C. M. Lee, E. P. Reddy, and S. K. P. Kung, "A novel role of the scaffolding protein JLP in tuning CD40-induced activation of dendritic cells," Immunobiology, vol. 218, no. 6, pp. 835-843, 2013.

[15] Y. Zhang, X. Li, S. Fang et al., "Peroxisome proliferatoractivated receptor $\gamma$ agonist suppresses mast cell maturation and induces apoptosis," Molecular Medicine Reports, vol. 16, no. 2, pp. 1793-1800, 2017.

[16] H. Y. Lee, J. Hur, I. K. Kim et al., "Effect of nintedanib on airway inflammation and remodeling in a murine chronic asthma model," Experimental Lung Research, vol. 43, no. 4-5, pp. 187196, 2017.

[17] A. Le Floc'h, J. Allinne, K. Nagashima et al., "Dual blockade of IL-4 and IL-13 with dupilumab, an IL-4R $\alpha$ antibody, is required to broadly inhibit type 2 inflammation," Allergy, vol. 75, pp. 466-476, 2020.

[18] T. Iinuma, Y. Okamoto, Y. Morimoto et al., "Pathogenicity of memory Th2 cells is linked to stage of allergic rhinitis," Allergy, vol. 73, pp. 479-489, 2017.

[19] T. Z. Zhang, F. U. Qiang, C. H. E. N. Tong, and M. A. Shi-Ping, "Anti-asthmatic effects of oxymatrine in a mouse model of allergic asthma through regulating CD40 signaling," Chinese Journal of Natural Medicines, vol. 13, no. 5, pp. 368-374, 2015.

[20] Z. Zietkowski, R. Skiepko, M. M. Tomasiak, and A. BodzentaLukaszyk, "Soluble CD40 ligand and soluble P-selectin in allergic asthma patients during exercise-induced bronchoconstriction," Journal of Investigational Allergology \& Clinical Immunology, vol. 18, no. 4, pp. 272-278, 2008.

[21] G. U. Hong, B. S. Park, J. W. Park, S. Y. Kim, and J. Y. Ro, "IgE production in CD40/CD40L cross-talk of B and mast cells and mediator release via TGase 2 in mouse allergic asthma," Cellular Signalling, vol. 25, no. 6, pp. 1514-1525, 2013.

[22] J. H. Park, H. S. Chang, C. S. Park et al., "Association analysis of CD40 polymorphisms with asthma and the level of serum total IgE," American Journal of Respiratory and Critical Care Medicine, vol. 175, no. 8, pp. 775-782, 2007.

[23] H. Hattori, M. Okano, S. Kariya, K. Nishizaki, and A. R. Satoskar, "Signals through CD40 play a critical role in the pathophysiology of Schistosoma mansoni egg antigen- 
induced allergic rhinitis in mice," American Journal of Rhinology, vol. 20, no. 2, pp. 165-169, 2006.

[24] H. Y. Jen, Y. H. Yang, B. L. Chiang, and Y. H. Chuang, "Upregulated interleukin-21 receptor on B cells associated with the downregulation of IgE in patients with allergic rhinitis," Journal of Interferon \& Cytokine Research, vol. 35, no. 1, pp. 42-48, 2015.

[25] M. Suzuki, M. Yokota, S. Ozaki, and Y. Nakamura, “A novel allergen-specific therapy with regulatory $\mathrm{T}$ cells induced by CD40-silenced dendritic cells," Asian Pacific Journal of Allergy and Immunology, vol. 37, no. 4, pp. 240-248, 2019.

[26] C. Senn, C. Hangartner, S. Moes, D. Guerini, and K. G. Hofbauer, "Central administration of small interfering RNAs in rats: a comparison with antisense oligonucleotides," European Journal of Pharmacology, vol. 522, no. 1-3, pp. 30-37, 2005.

[27] M. Suzuki, X. Zheng, X. Zhang et al., "Novel vaccination for allergy through gene silencing of CD40 using small interfering RNA," Journal of Immunology, vol. 180, no. 12, pp. 8461-8469, 2008.

[28] M. Suzuki, X. Zheng, X. Zhang et al., "Inhibition of allergic responses by CD40 gene silencing," Allergy, vol. 64, no. 3, pp. 387-397, 2009.

[29] M. Suzuki, X. Zheng, X. Zhang et al., “A novel allergen-specific therapy for allergy using CD40-silenced dendritic cells," The Journal of Allergy and Clinical Immunology, vol. 125, no. 3, pp. 737-743.e6, 2010.

[30] C. Pilette, M. R. Jacobson, C. Ratajczak et al., "Aberrant dendritic cell function conditions Th2-cell polarization in allergic rhinitis," Allergy, vol. 68, no. 3, pp. 312-321, 2013.

[31] A. Froidure, C. Shen, and C. Pilette, "Dendritic cells revisited in human allergic rhinitis and asthma," Allergy, vol. 71, no. 2, pp. 137-148, 2016.

[32] N. Novak, J. P. Allam, H. Betten, J. Haberstok, and T. Bieber, "The role of antigen presenting cells at distinct anatomic sites: they accelerate and they slow down allergies," Allergy, vol. 59, no. 1, pp. 5-14, 2004.

[33] T. Ito, M. Yang, Y. H. Wang et al., "Plasmacytoid dendritic cells prime IL-10-producing $\mathrm{T}$ regulatory cells by inducible costimulator ligand," The Journal of Experimental Medicine, vol. 204, no. 1, pp. 105-115, 2007.

[34] J. A. Portillo, J. A. Greene, I. Schwartz, M. C. Subauste, and C. S. Subauste, "Blockade of CD40-TRAF2,3 or CD40TRAF6 is sufficient to inhibit pro-inflammatory responses in non-haematopoietic cells," Immunology, vol. 144, no. 1, pp. 21-33, 2015.

[35] C. Barthels, A. Ogrinc, V. Steyer et al., "CD40-signalling abrogates induction of ROR $\gamma \mathrm{t}+$ Treg cells by intestinal CD103+ DCs and causes fatal colitis," Nature Communications, vol. 8, no. 1, article 14715, 2017.

[36] F. Xu, S. Yu, M. Qin et al., "Hydrogen-rich saline ameliorates allergic rhinitis by reversing the imbalance of Th1/Th2 and up-regulation of CD4+CD25+Foxp3+regulatory T cells, interleukin-10, and membrane-bound transforming growth factor$\beta$ in guinea pigs," Inflammation, vol. 41, no. 1, pp. 81-92, 2018.

[37] W. Wang, T. Jiang, Z. Zhu, J. Cui, L. Zhu, and Z. Ma, "Dexamethasone suppresses allergic rhinitis and amplifies CD4(+) Foxp3(+) regulatory T cells in vitro," Int Forum Allergy Rhinol., vol. 5, no. 10, pp. 900-906, 2015.

[38] L. Tan, J. Ou, Z. Tao, Y. Kong, and Y. Xu, "Neonatal immune state is influenced by maternal allergic rhinitis and associated with regulatory T cells," Allergy Asthma Immunol Res., vol. 9, no. 2, pp. 133-141, 2017.

[39] W. Liu, H. Ouyang, Q. Zeng, R. Luo, and G. Lu, "Decreased Treg-derived miR-181a and miR-155 correlated with reduced number and function of Treg cells in allergic rhinitis children," European Archives of Oto-Rhino-Laryngology, vol. 276, no. 4, pp. 1089-1094, 2019.

[40] A. Jo, S. H. Lee, D. Y. Kim et al., "Mast cell-derived plasminogen activator inhibitor type 1 promotes airway inflammation and remodeling in a murine model of asthma," The Journal of Allergy and Clinical Immunology, vol. 142, no. 1, pp. 294297.e5, 2018.

[41] A. K. Mehta, T. Doherty, D. Broide, and M. Croft, "Tumor necrosis factor family member LIGHT acts with IL- $1 \beta$ and TGF- $\beta$ to promote airway remodeling during rhinovirus infection," Allergy, vol. 73, no. 7, pp. 1415-1424, 2018.

[42] J. G. Liu, M. Q. Wang, X. H. Zhu, Y. H. Liu, and J. Y. Cai, "Microvascular remodeling of nasal mucosa in allergic rhinitis induced by an allergen in Sprague-Dawley rats," Genetics and Molecular Research, vol. 14, no. 3, pp. 11624-11630, 2015.

[43] Z. Y. Chen, S. H. Zhou, Q. F. Zhou, and H. B. Tang, "Inflammation and airway remodeling of the lung in guinea pigs with allergic rhinitis," Experimental and Therapeutic Medicine, vol. 14, no. 4, pp. 3485-3490, 2017.

[44] J. Corren and A. Togias, "Remodeling in allergic rhinitis. Adding new data to an old debate," American Journal of Respiratory and Critical Care Medicine, vol. 192, no. 12, pp. 1403-1404, 2015.

[45] A. O. Eifan, N. T. Orban, M. R. Jacobson, and S. R. Durham, "Severe persistent allergic rhinitis. Inflammation but no histologic features of structural upper airway remodeling," American Journal of Respiratory and Critical Care Medicine, vol. 192, no. 12, pp. 1431-1439, 2015. 